\title{
Optimization of the electronic band structure and the lattice thermal conductivity of solid solutions according to simple calculations: a canonical example of the $\mathrm{Mg}_{2} \mathrm{Si}_{1-x-y} \mathbf{G e}_{x} \mathrm{Sn}_{y}$ ternary solid solution
}

Kang Yin, ${ }^{\dagger}$ Xianli Su, ${ }^{\dagger *}$ Yonggao Yan $^{\dagger}$, Yonghui You ${ }^{\dagger}$, Qiang Zhang ${ }^{\dagger}$, Ctirad Uher ${ }^{\star}$, Mercouri G. Kanatzidis $^{\xi}$ and Xinfeng Tang ${ }^{\dagger *}$

${ }^{\dagger}$ State Key Laboratory of Advanced Technology for Materials Synthesis and Processing, Wuhan University of Technology, Wuhan 430070, China

$\$$ Department of Physics, University of Michigan, Ann Arbor, Michigan48109, USA

${ }^{\xi}$ Department of Chemistry, Northwestern University, Evanston, Illinois 60208, USA

Supporting Information

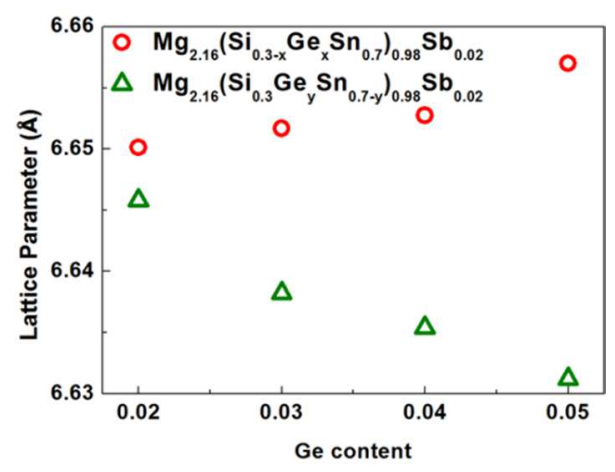

Figure SI1. The calculated lattice parameter for $\mathrm{Mg}_{2.16}\left(\mathrm{Si}_{0.3-x} \mathrm{Ge}_{x} \mathrm{Sn}_{0.7}\right)_{0.98} \mathrm{Sb}_{0.02}$ and $\mathrm{Mg}_{2.16}\left(\mathrm{Si}_{0.3} \mathrm{Ge}_{y} \mathrm{Sn}_{0.7-}\right.$ $\left.{ }_{y}\right)_{0.98} \mathrm{Sb}_{0.02}(0 \leq x, y \leq 0.05)$ as a function of Ge content.

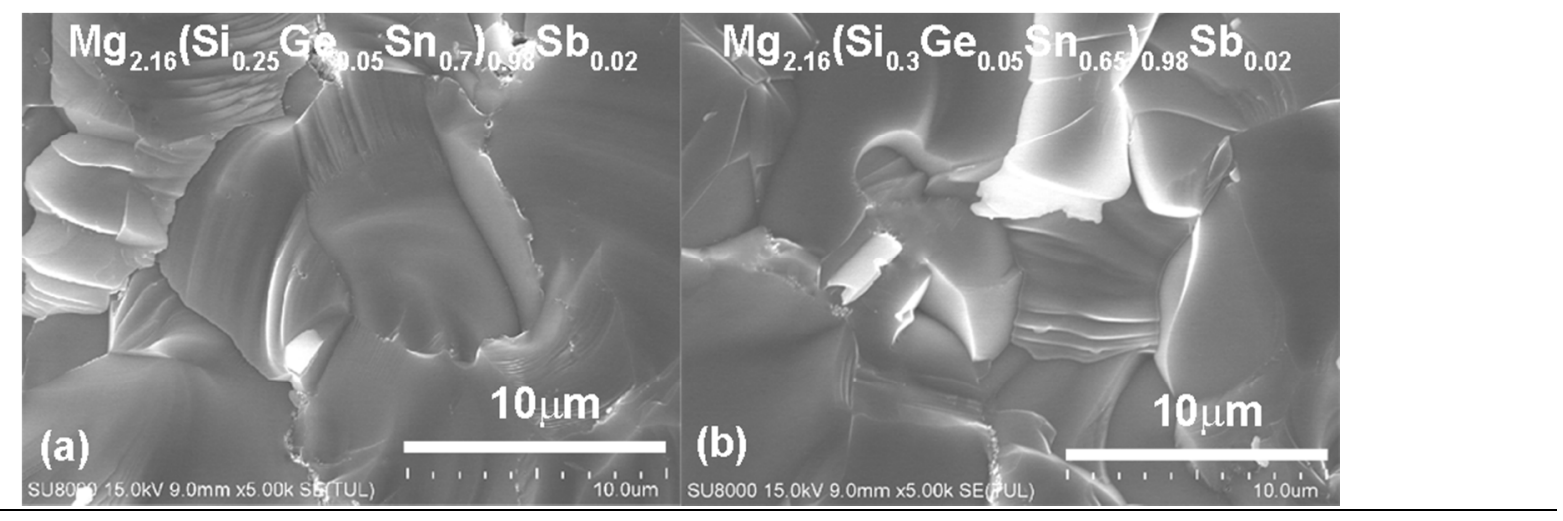


Figure SI2. The microstructure of $\mathrm{Mg}_{2.16}\left(\mathrm{Si}_{0.3-x} \mathrm{Ge}_{x} \mathrm{Sn}_{0.7}\right)_{0.98} \mathrm{Sb}_{0.02}(x=0.05)$ and $\mathrm{Mg}_{2.16}\left(\mathrm{Si}_{0.3} \mathrm{Ge}_{y} \mathrm{Sn}_{0.7-}\right.$ $\left.{ }_{y}\right)_{0.98} \mathrm{Sb}_{0.02}(y=0.05)$. No nanostructures were observed.
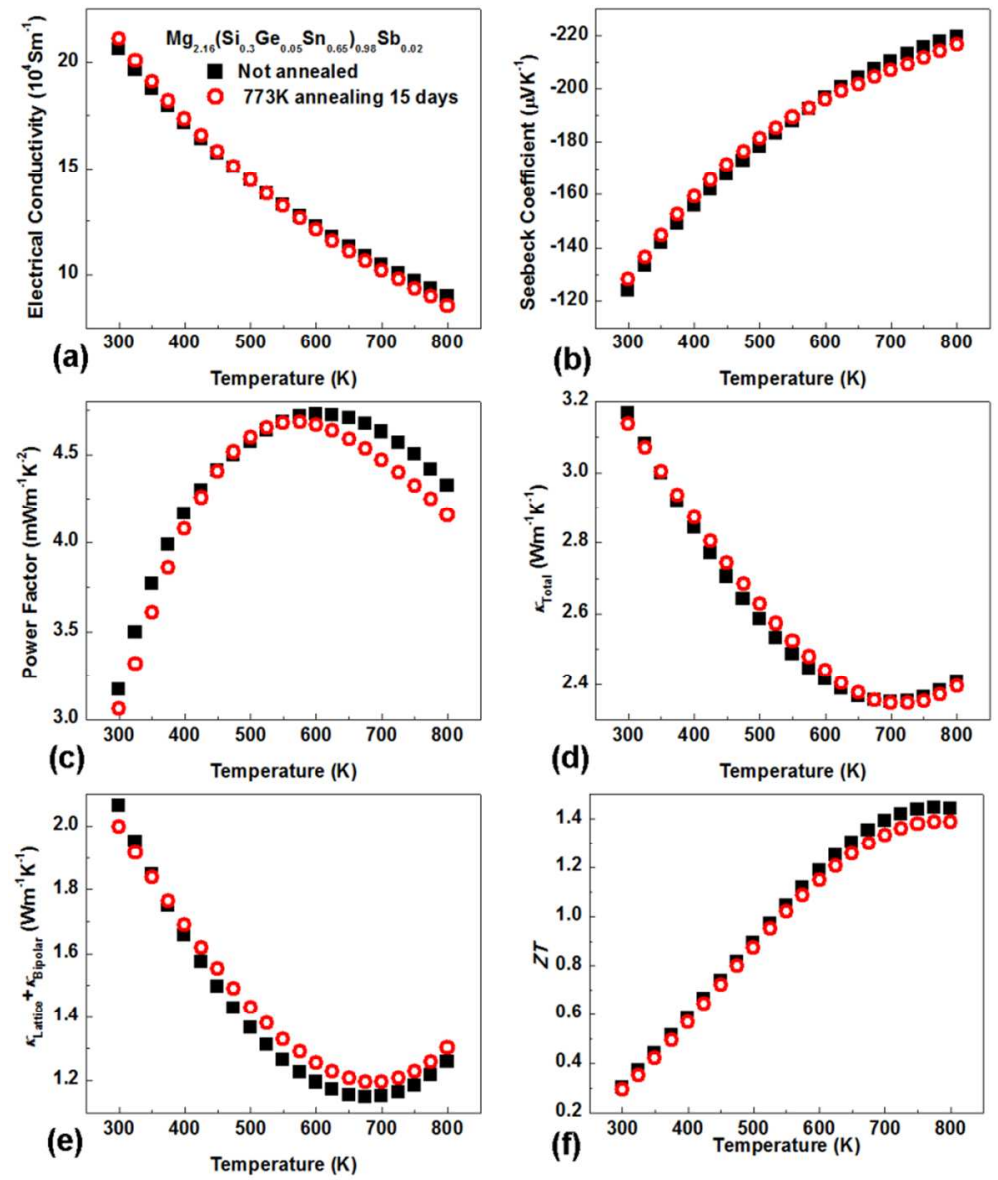

Figure SI3.: The thermoelectric properties of $\mathrm{Mg}_{2.16}\left(\mathrm{Si}_{0.3} \mathrm{Ge}_{0.05} \mathrm{Sn}_{0.65}\right)_{0.98} \mathrm{Sb}_{0.02}$ before and after annealing at $773 \mathrm{~K}$ with BN coatings in air. (a) the electrical conductivity $\sigma$, (b) the Seebeck coefficient $S$; (c) the power factor $P F$; (d) the total thermal conductivity $\kappa_{\text {Total }}$; (e) the sum of the lattice thermal conductivity and the bipolar part $\kappa_{\text {Lattice }}+\kappa_{\text {Bipolar; }}$, and (f) the dimensionless figure of merit $Z T$.

If only Umklapp and point defect phonon scattering processes are taken into consideration, the ratio of $\kappa_{\mathrm{L}}$ of the crystal with disorder to that without disorder $\kappa_{\mathrm{L}}^{\mathrm{P}}$ can be expressed as:

$$
\frac{\kappa_{\mathrm{L}}}{\kappa_{\mathrm{L}}^{\mathrm{P}}}=\frac{\tan ^{-1} u}{u}, \quad u^{2}=\frac{\pi^{2} \theta_{\mathrm{D}} \Omega \kappa_{\mathrm{L}}^{\mathrm{P}} \Gamma_{\text {expt }}}{h v^{2}}
$$

where $u, \Omega, \theta_{\mathrm{D}}, h, v$, and $\Gamma_{\text {expt }}$ are the disorder scaling parameter, the average volume per atom, the Debye temperature (taken as $435 \mathrm{~K}$, as reported by G. Jiang et al. ${ }^{1}$ ), the Planck constant, the average lattice sound velocity ((taken as $5300 \mathrm{~ms}^{-1}$, as reported by W. Liu et al. $\left.{ }^{2}\right)$,), and the experimental disorder scattering parameter, respectively.

Usually, researchers treat $\Gamma_{\text {expt }}=\Gamma_{\text {calc }}=\Gamma_{\mathrm{M}}+\Gamma_{\mathrm{S}},{ }^{1,3,4}$ where $\Gamma_{\mathrm{M}}$ and $\Gamma_{\mathrm{S}}$ are the scattering parameters due to mass and strain field fluctuations, respectively. If the $k^{\text {th }}$ atom of the $i^{\text {th }}$ sublattice has mass $M_{i}^{k}$, 
radius $r_{i}^{k}$, and fractional occupation $f_{i}^{k}$, so the average mass and radius of atoms on the ith sublattice are:

$$
\overline{M_{i}}=\sum_{k} f_{i}^{k} M_{i}^{k}, \quad \bar{r}_{i}=\sum_{k} f_{i}^{k} r_{i}^{k}
$$

And the average atomic mass of the compound is:

$$
\overline{\bar{M}}=\frac{\sum_{i=1}^{n} c_{i} \overline{M_{i}}}{\sum_{i=1}^{n} c_{i}}
$$

For three different atoms on each of the $i^{\text {th }}$ sublattices, i. e., $k=1,2,3$ for $\mathrm{Si}$, Ge and $\mathrm{Sn}$ atoms, and $n=3, c_{1}=c_{2}=c_{3}=1$, using $f_{i}^{1}+f_{i}^{2}+f_{i}^{3}=1$ and $\overline{M_{i}}=f_{i}^{1} M_{i}^{1}+f_{i}^{2} M_{i}^{2}+f_{i}^{3} M_{i}^{3}$, we can get

$$
\begin{gathered}
\Gamma_{\mathrm{M}}=\frac{\sum_{i=1}^{n} c_{i}\left(\frac{\overline{M_{i}}}{\overline{\bar{M}}}\right)^{2}\left[f_{i}^{1} f_{i}^{2}\left(\frac{M_{i}^{1}-M_{i}^{2}}{\overline{M_{i}}}\right)^{2}+f_{i}^{1} f_{i}^{3}\left(\frac{M_{i}^{1}-M_{i}^{3}}{M_{i}}\right)^{2}+f_{i}^{2} f_{i}^{3}\left(\frac{M_{i}^{2}-M_{i}^{3}}{\bar{M}_{i}}\right)^{2}\right]}{\sum_{i=1}^{n} c_{i}} \\
\Gamma_{\mathrm{S}}=\frac{\sum_{i=1}^{n} c_{i} \varepsilon_{i}\left(\frac{\overline{M_{i}}}{\bar{M}}\right)^{2}\left[f_{i}^{1} f_{i}^{2}\left(\frac{r_{i}^{1}-r_{i}^{2}}{\overline{r_{i}}}\right)^{2}+f_{i}^{1} f_{i}^{3}\left(\frac{r_{i}^{1}-r_{i}^{3}}{\overline{r_{i}}}\right)^{2}+f_{i}^{2} f_{i}^{3}\left(\frac{r_{i}^{2}-r_{i}^{3}}{r_{i}}\right)^{2}\right]}{\sum_{i=1}^{n} c_{i}}
\end{gathered}
$$

And the value of $\varepsilon_{\mathrm{S}}$ can be figured out by solving equations (1)-(5), as other parameters can be obtained by experiments or references.

1. Jiang, G.; He, J.; Zhu, T.; Fu, C.; Liu, X.; Hu, L.; Zhao, X., High Performance $\mathrm{Mg}_{2}(\mathrm{Si}, \mathrm{Sn})$ Solid Solutions: a Point Defect Chemistry Approach to Enhancing Thermoelectric Properties. Adv. Func. Mater. 2014, 24, (24), 3776-3781.

2. $\quad$ Liu, W.; Chi, H.; Sun, H.; Zhang, Q.; Yin, K.; Tang, X.; Zhang, Q.; Uher, C., Advanced thermoelectrics governed by a single parabolic band: $\mathrm{Mg}_{2} \mathrm{Si}_{0.3} \mathrm{Sn}_{0.7}$, a canonical example. Phys. Chem. Chem. Phys. 2014, 16, (15), 6893-6897.

3. Abeles, B., Lattice Thermal Conductivity of Disordered Semiconductor Alloys at High Temperatures. Phys. Rev. 1963, 131, (5), 1906-1911.

4. $\quad$ Yang, J.; Meisner, G. P.; Chen, L., Strain field fluctuation effects on lattice thermal conductivity of ZrNiSn-based thermoelectric compounds. Appl. Phys. Lett. 2004, 85, (7), 1140-1142. 